\title{
Adverse Event MedDRA High Level Group Term Code
}

National Cancer Institute

\section{Source}

National Cancer Institute. Adverse Event MedDRA High Level Group Term Code. NCI Thesaurus. Code C117379.

A coded value specifying the high level group term for the adverse event from the Medical Dictionary for Regulatory Activities (MedDRA). 